\title{
On the Dynamics of a Two-Strain Influenza Model with Isolation
}

\author{
F. Chamchod ${ }^{1,2 *}$, N. F. Britton ${ }^{2}$ \\ ${ }^{1}$ Department of Mathematics, University of Miami, Coral Gables, Miami, FL 33124-4250, USA \\ ${ }^{2}$ Centre for Mathematical Biology, Department of Mathematical Sciences, University of Bath, Bath \\ BA2 7AY, UK.
}

\begin{abstract}
Influenza has been responsible for human suffering and economic burden worldwide. Isolation is one of the most effective means to control the disease spread. In this work, we incorporate isolation into a two-strain model of influenza. We find that whether strains of influenza die out or coexist, or only one of them persists, it depends on the basic reproductive number of each influenza strain, cross-immunity between strains, and isolation rate. We propose criteria that may be useful for controlling influenza. Furthermore, we investigate how effective isolation is by considering the host's mean age at infection and the invasion rate of a novel strain. Our results suggest that isolation may help to extend the host's mean age at infection and reduce the invasion rate of a new strain. When there is a delay in isolation, we show that it may lead to more serious outbreaks as compared to no delay.
\end{abstract}

Keywords and phrases: isolation, influenza, strain dynamics

Mathematics Subject Classification: 92B05

\section{Introduction}

The emergence and reemergence of multi-strain infectious diseases driven by mutations has been known as a cause of death worldwide. Pathogen mutations are found commonly in many infectious diseases such as: 1) measles [1], 2) hepatitis B [2-4], 3) HIV [5], 4) West Nile virus [6], 5) pertussis [7, 8], 6) malaria [9], and 7) influenza [10,11]. In this work, we are particularly interested in the dynamics of influenza. Influenza is an RNA virus which is separated into three types A, B, and C. Influenza A is the most common one in human and animal populations. On the influenza surface, there are two important proteins, hemagglutinin (HA) and neuraminidase (NA), that change significantly over time.

Two mechanisms of influenza viruses, shift and drift processes, allow them to escape the recognition of the host immune system. Antigenic shift generates a new subtype by drastically changing protein molecules (HA and NA) on a viral surface, while antigenic drift continually changes the protein molecules by point mutations to generate a new strain. In host, cross-immunity plays an important role against the escape mutant $[12,13]$. Cross-immunity is high between similar strains and very low when they are genetically different, two strains from different subtypes for instance [14,15]. Cross-immunity may act in different ways such as reducing susceptibility when hosts get infected with one strain and become

\footnotetext{
${ }^{*}$ Corresponding author. E-mail: fchamchod@gmail.com
} 
less susceptible to other related strains, reducing transmissibility during subsequent infections with similar strains from previous infection, and polarizing immunity when only some individuals acquire total immunity to related strains from previous infection while others gain no immunity.

Mathematical models of multi-strain diseases have fruitfully been used to study the dynamics of the pathogens in a host population [16-29]. By considering the models relating to cross-immunity, they can be separated into many types, for instance a moving frame in immunity spaces [30,31], a season-to-season drift [25, 26], a history-based framework [18], and a status-based framework [21,22]. This work is based on the latter type, where susceptible individuals are categorized by their current immune status instead of their immune memory.

Controlling diseases is an important means for public health to reduce the cost arising from the spread of the diseases. Several control strategies are available for influenza such as isolation, vaccination, and the use of treatments. Here, we only concentrate on isolation. Isolation is applied to infected individuals so as to prevent them from further contacts and subsequent transmissions to other individuals. It is primarily used for controlling the disease when it suddenly emerges or reemerges. A successful example is the isolation of those infected with SARS during 2003-2004. However, the disadvantages of this strategy are the difficulty of detecting infected individuals and the cost of isolation. Although many models for multiple-strain diseases have been fruitfully developed, models including isolation are very limited $[32,33]$.

In this work, we study the dynamics of a host population when two strains of influenza are cocirculating. Inspired by the current use of isolation, we propose a new model to investigate how isolation may help to control influenza by considering threshold conditions, the host's mean age at infection, the invasion rate of a new strain, and delay in isolation.

\section{Model formulation}

We here propose a model of isolation. The model is based on a status-based framework by [22]. The model assumes polarized susceptibility, i.e. each individual is either completely immune or completely susceptible to each strain of a disease. Immediately on infection with a strain of a disease, an individual is assumed to become immune to that strain with probability 1 and immune to related strains with reduced probabilities. For a disease with two co-circulating strains, each individual, whether infected or not, has one of four possible immune repertoires $\emptyset,\{1\},\{2\},\{1,2\}$. We assume that an individual with immune repertoire $\emptyset$ has probability $\sigma$ of acquiring immunity to strain 2 (as well as strain 1 ) on infection with strain 1, and the same probability $\sigma$ of acquiring immunity to strain 1 on infection with strain 2 . Following [22], we assume further that an individual with immune repertoire $\{1\}$ also has probability $\sigma$ of acquiring immunity to strain 2 on contact with an individual infected with strain 1 even though infection with strain 1 is impossible, and similarly when an individual with immune repertoire $\{2\}$ comes into contact with an individual infected with strain 2. These assumptions allow us to write down a system of four equations for four classes of hosts: susceptible to strain $1, S_{1}$ (whether with immune repertoire $\emptyset$ or $\{2\}$ and whether or not infected with strain 2); infected with strain $1, I_{1}$; susceptible to strain $2, S_{2}$; and infected with strain $2, I_{2}$. Note that these classes are not mutually exclusive, and that some individuals do not belong to any of them. We assume that the a recovery rate from each strain is the same and denote it by $\nu_{0}$, but that each strain has a different rate of transmission denoted by $\beta_{1}$ and $\beta_{2}$.

Because of similar symptoms and the difficulty of clinically distinguishing a viral strain, we assume that rate at which infected individuals are detected and removed to isolation $\left(q_{0}\right)$ is the same for strain 1 and strain 2. Removing such individuals to isolation does not change their immune status and they return to the same $S_{i}$ class after recovery, so (on the assumption that there are few individuals in isolation at any one time) the equations for $S_{1}$ and $S_{2}$ are unchanged. We assume that individuals leave the isolation class when they recover, hence, at rate $\nu_{0}$. 
The model takes the following form:

$$
\begin{aligned}
& S_{1}^{\prime}(t)=\mu_{0} N-\mu_{0} S_{1}-\beta_{1} S_{1} I_{1}-\sigma \beta_{2} S_{1} I_{2}, \\
& I_{1}^{\prime}(t)=\beta_{1} S_{1} I_{1}-\left(\nu_{0}+\mu_{0}+q_{0}\right) I_{1}, \\
& S_{2}^{\prime}(t)=\mu_{0} N-\mu_{0} S_{2}-\beta_{2} S_{2} I_{2}-\sigma \beta_{1} S_{2} I_{1}, \\
& I_{2}^{\prime}(t)=\beta_{2} S_{2} I_{2}-\left(\nu_{0}+\mu_{0}+q_{0}\right) I_{2}, \\
& Q^{\prime}(t)=q_{0}\left(I_{1}+I_{2}\right)-\nu_{0} Q .
\end{aligned}
$$

Note that this model can be derived from the status-based model presented by [21] and simplified by the assumptions in [22]. We consider the status-based model with isolation for two strains of influenza as follows:

$$
\begin{aligned}
\dot{S}_{\phi}= & \mu N-\mu S_{\phi}-C(\phi,\{1\}, 1) \Lambda_{1} S_{\phi}-C(\phi,\{1,2\}, 1) \Lambda_{1} S_{\phi}- \\
& C(\phi,\{2\}, 2) \Lambda_{2} S_{\phi}-C(\phi,\{1,2\}, 2) \Lambda_{2} S_{\phi}, \\
\dot{S}_{\{1\}}= & C(\phi,\{1\}, 1) \Lambda_{1} S_{\phi}-C(\{1\},\{1,2\}, 2) \Lambda_{2} S_{\{1\}}- \\
& C(\{1\},\{1,2\}, 1) \Lambda_{1} S_{\{1\}}-\mu S_{\{1\}}, \\
\dot{S}_{\{2\}}= & C(\phi,\{2\}, 2) \Lambda_{2} S_{\phi}-C(\{2\},\{1,2\}, 1) \Lambda_{1} S_{\{2\}}- \\
& C(\{2\},\{1,2\}, 2) \Lambda_{2} S_{\{2\}}-\mu S_{\{2\}}, \\
\dot{S}_{\{1,2\}}= & C(\phi,\{1,2\}, 1) \Lambda_{1} S_{\phi}+C(\phi,\{1,2\}, 2) \Lambda_{2} S_{\phi}+ \\
& C(\{1\},\{1,2\}, 2) \Lambda_{2} S_{\{1\}}+C(\{2\},\{1,2\}, 1) \Lambda_{1} S_{\{2\}}-\mu S_{\{1,2\}}, \\
\dot{I}_{1}= & \Lambda_{1}\left(S_{\phi}+S_{\{2\}}\right)-\left(\mu+\nu_{1}+q_{1}\right) I_{1}, \\
\dot{I}_{2}= & \Lambda_{2}\left(S_{\phi}+S_{\{1\}}\right)-\left(\mu+\nu_{2}+q_{2}\right) I_{2}, \\
\dot{Q}_{1}= & q_{1} I_{1}-\kappa_{1} Q_{1}, \\
\dot{Q}_{2}= & q_{2} I_{2}-\kappa_{2} Q_{2},
\end{aligned}
$$

where $\mathcal{J} \subseteq\{1,2\}, \Lambda_{i}=\beta_{i} I_{i}, S_{\mathcal{J}}$ represents the number of hosts that are completely immune to the strains in the set $\mathcal{J}, I_{i}$ the number of infectious individuals to strain $i, Q_{i}$ the number of isolated individuals, previously infected with strain $i$, and $C(\mathcal{L}, \mathcal{J}, i)$ the probability that hosts recover to a state $\mathcal{J}$, having started in state $\mathcal{L}$ and been infected by strain $i$. A flow diagram is shown in Figure 2 and other parameters can be found in Table 1.

In this model, we assume that rates at which individuals infected with strain 1 and strain 2 are detected and removed to isolation are $q_{1}$ and $q_{2}$, respectively. We assume that isolated individuals are removed at rate $\kappa_{1}$ for strain 1 and $\kappa_{2}$ for strain 2, whenever they recover [34]. Note that removing individuals to isolation does not change their immune status, so they return to the same $S_{i}$ class after recovery (see Figure 2). For simplicity, we now assume that rates of detecting individuals infected with strain 1 and 2 are equivalent $\left(q_{1}=q_{2}=q_{0}\right)$. Also, individuals have the same rate of recovery from both strains $\left(\nu_{1}=\nu_{2}=\nu_{0}\right)$ and rate of removing individuals from isolation equals rate of recovery $\left(\kappa_{1}=\kappa_{2}=\nu_{0}\right)$.

The polarized immunity is assumed which means that cross-immunity acts to render some hosts totally immune while other hosts gain nothing from infections. Also, the reduced-transmission of cross-immunity is assumed. By assuming that cross-immunity is conferred by exposure even if immunity prevents the full disease from developing, this allows all hosts to have the same chance of gaining immunity to a strain whatever their current immune status is. The system (2.2) can be reduced such that only one variable is needed to explain the host with respect to each strain. Since $S_{\{1,2\}}$ does not appear in other equations, it can be omitted from the system. We introduce a new variable $Q$ as the total number of isolated individuals previously infected with both strains of influenza, $Q=Q_{1}+Q_{2}$. Let $S_{1}=S_{\phi}+S_{\{2\}}$ and $S_{2}=S_{\phi}+S_{\{1\}}$. Hence, by the fact that $\sum_{\mathcal{J}} C(\mathcal{K}, \mathcal{J}, i)=1$ :

$$
\dot{S}_{1}=\mu N-\mu S_{1}-\Lambda_{1} S_{1}-C(\phi,\{1,2\}, 2) \Lambda_{2} S_{\phi}-C(\{2\},\{1,2\}, 2) \Lambda_{2} S_{\{2\}}
$$

Define $C(\phi,\{1,2\}, 2)=C(\{2\},\{1,2\}, 2)=\sigma_{12}$, which is the chance that an infection by strain 2 gives immunity to strain 1 . Hence,

$$
\dot{S}_{1}=\mu N-\mu S_{1}-\Lambda_{1} S_{1}-\sigma_{12} \Lambda_{2} S_{1} .
$$

The equations for $S_{2}$ can be obtained in the similar way. We further assume that $\sigma_{12}=\sigma_{21}$, for simplicity. Therefore, the system (2.2) becomes the system (2.1) 


\section{Analysis}

We normalise the system (2.1) by introducing the new variables and parameters as follows:

$$
\begin{gathered}
s_{1}=\frac{S_{1}}{N}, i_{1}=\frac{I_{1}}{N}, s_{2}=\frac{S_{2}}{N}, i_{2}=\frac{I_{2}}{N}, q=\frac{Q}{N}, \\
T=\left(\mu_{0}+\nu_{0}+q_{0}\right) t, \mu=\frac{\mu_{0}}{\mu_{0}+\nu_{0}+q_{0}}, \theta=\frac{q_{0}}{\mu_{0}+\nu_{0}+q_{0}}, \nu=\frac{\nu_{0}}{\mu_{0}+\nu_{0}+q_{0}},
\end{gathered}
$$

and the basic reproductive numbers for strain 1 and strain 2 in both interventions are

$$
R_{1 q}=\frac{\beta_{1} N}{\mu_{0}+\nu_{0}+q_{0}} \text { and } R_{2 q}=\frac{\beta_{2} N}{\mu_{0}+\nu_{0}+q_{0}} .
$$

Note that, for strain $i$, a single infectious individual introduced to an entirely susceptible population makes $\beta_{i} N$ contacts in unit time with susceptible individuals during its infectious period, which is $1 /\left(\mu_{0}+\nu_{0}+q_{0}\right)$. We also remark here that $R_{1 q}$ and $R_{2 q}$ can be written in terms of $R_{1}\left(\beta_{1} N /\left(\mu_{0}+\nu_{0}\right)\right)$ and $R_{2}\left(\beta_{2} N /\left(\mu_{0}+\right.\right.$ $\left.\nu_{0}\right)$ ), the basic reproductive numbers of strain 1 and 2 when isolation is absent as

$$
R_{1 q}=(1-\theta) R_{1} \text { and } R_{2 q}=(1-\theta) R_{2} .
$$

The model is now in the following form:

$$
\begin{aligned}
& s_{1}^{\prime}(T)=\mu\left(1-s_{1}\right)-R_{1 q} s_{1} i_{1}-\sigma R_{2 q} s_{1} i_{2}, \\
& i_{1}^{\prime}(T)=R_{1 q} s_{1} i_{1}-i_{1}, \\
& s_{2}^{\prime}(T)=\mu\left(1-s_{2}\right)-R_{2 q} s_{2} i_{2}-\sigma R_{1 q} s_{2} i_{1}, \\
& i_{2}^{\prime}(T)=R_{2 q} s_{2} i_{2}-i_{2}, \\
& q^{\prime}(T)=\theta\left(i_{1}+i_{2}\right)-\nu q .
\end{aligned}
$$

\subsection{Steady states}

By setting the right-hand sides of the system (3.1), we have four steady states:

1. The disease-free steady state

$$
P_{q}^{0}=\left(s_{1 q}^{0}, i_{1 q}^{0}, s_{2 q}^{0}, i_{2 q}^{0}, q_{q}^{0}\right)=(1,0,1,0,0) .
$$

2. The single-strain steady state of strain 1

$$
P_{q}^{1}=\left(s_{1 q}^{1}, i_{1 q}^{1}, s_{2 q}^{1}, i_{2 q}^{1}, q_{q}^{1}\right)=\left(\frac{1}{R_{1 q}}, \frac{\mu}{R_{1 q}}\left(R_{1 q}-1\right), \frac{1}{1+\sigma\left(R_{1 q}-1\right)}, 0, \frac{\theta \mu}{\nu R_{1 q}}\left(R_{1 q}-1\right)\right)
$$

which is positive if and only if

$$
R_{1 q}>1
$$

3. The single-strain steady state of strain 2

$$
P_{q}^{2}=\left(s_{1 q}^{2}, i_{1 q}^{2}, s_{2 q}^{2}, i_{2 q}^{2}, q_{q}^{2}\right)=\left(\frac{1}{1+\sigma\left(R_{2 q}-1\right)}, 0, \frac{1}{R_{2 q}}, \frac{\mu}{R_{2 q}}\left(R_{2 q}-1\right), \frac{\theta \mu}{\nu R_{2 q}}\left(R_{2 q}-1\right)\right)
$$

which is positive if and only if

$$
R_{2 q}>1 \text {. }
$$

4. The coexistent steady state

$$
P_{q}^{*}=\left(s_{1 q}^{*}, i_{1 q}^{*}, s_{2 q}^{*}, i_{2 q}^{*}, q_{q}^{*}\right)
$$

where

$$
s_{1 q}^{*}=\frac{1}{R_{1 q}}, \quad i_{1 q}^{*}=\frac{\mu}{\left(1-\sigma^{2}\right) R_{1 q}}\left[\left(R_{1 q}-1\right)-\sigma\left(R_{2 q}-1\right)\right],
$$




$$
s_{2 q}^{*}=\frac{1}{R_{2 q}}, \quad i_{2 q}^{*}=\frac{\mu}{\left(1-\sigma^{2}\right) R_{2 q}}\left[\left(R_{2 q}-1\right)-\sigma\left(R_{1 q}-1\right)\right]
$$

and

$$
q_{q}^{*}=\frac{\theta \mu}{\left(1-\sigma^{2}\right) \nu R_{1 q} R_{2 q}}\left[R_{2 q}\left(R_{1 q}-1\right)+R_{1 q}\left(R_{2 q}-1\right)-\sigma\left[R_{1 q}\left(R_{1 q}-1\right)+R_{2 q}\left(R_{2 q}-1\right)\right]\right],
$$

which is positive if and only if both of the following conditions hold

$$
\left(R_{1 q}-1\right)-\sigma\left(R_{2 q}-1\right)>0
$$

and

$$
\left(R_{2 q}-1\right)-\sigma\left(R_{1 q}-1\right)>0 .
$$

Note that from these two conditions, $q_{q}^{*}$ is consequently positive. Also note that the steady states when there is no isolation can be found by setting $\theta$ equaling to zero and they are parallel to the steady states here.

\subsection{Stability analysis}

Theorem 3.1. Stability conditions for each steady state are as follows:

1. The disease-free steady state is stable if and only if $R_{1 q}<1$ and $R_{2 q}<1$, or $\theta>1-\frac{1}{R_{1}}$ and $\theta>1-\frac{1}{R_{2}}$.

2. The single-strain steady state of strain 1 is stable if and only if $R_{1 q}>1$ and $R_{2 q}-1-\sigma\left(R_{1 q}-1\right)<0$, or $\theta<1-\frac{1}{R_{1}}$ and $\left(R_{2}-1\right)-\sigma\left(R_{1}-1\right)<\left(R_{2}-\sigma R_{1}\right) \theta$.

3. The single-strain steady state of strain 2 is stable if and only if $R_{2 q}>1$ and $R_{1 q}-1-\sigma\left(R_{2 q}-1\right)<0$, or $\theta<1-\frac{1}{R_{2}}$ and $\left(R_{1}-1\right)-\sigma\left(R_{2}-1\right)<\left(R_{1}-\sigma R_{2}\right) \theta$.

4. The coexistent steady state is stable if and only if $R_{2 q}-1-\sigma\left(R_{1 q}-1\right)>0$ and $R_{1 q}-1-\sigma\left(R_{2 q}-1\right)>0$, or $\left(R_{2}-1\right)-\sigma\left(R_{1}-1\right)>\left(R_{2}-\sigma R_{1}\right) \theta$ and $\left(R_{1}-1\right)-\sigma\left(R_{2}-1\right)>\left(R_{1}-\sigma R_{2}\right) \theta$.

Proof. The stability conditions can be derived by considering the Jacobian matrix of the system (3.1) and using the Routh-Hurwitz criteria. Each steady state is stable if and only if all eigenvalues of the Jacobian matrix at the steady state have negative real parts. In case we cannot determine the eigenvalues of the Jacobian matrix explicitly, we consider whether the coefficients of the characteristic equation satisfy the Routh-Hurwitz criteria [35]. If the characteristic polynomial is of order 4,

$$
\lambda^{4}+a_{1} \lambda^{3}+a_{2} \lambda^{2}+a_{3} \lambda+a_{4}=0,
$$

the steady state is stable if and only if

$$
a_{1}>0, a_{3}>0, a_{4}>0, \text { and } a_{1} a_{2} a_{3}>a_{3}^{2}+a_{1}^{2} a_{4} .
$$

Since $Q$ does not appear in other differential equations in (3.1), it can be omitted from the system. The Jacobian matrix of the system (3.1) is

$$
J=\left[\begin{array}{lccr}
-\mu-R_{1 q} i_{1}-\sigma R_{2 q} i_{2} & -R_{1 q} s_{1} & 0 & -\sigma R_{2 q} s_{1} \\
R_{1 q} i_{1} & R_{1 q} s_{1}-1 & 0 & 0 \\
0 & -\sigma R_{1 q} s_{2} & -\mu-R_{2 q} i_{2}-\sigma R_{1 q} i_{1} & -R_{2 q} s_{2} \\
0 & 0 & R_{2 q} i_{2} & R_{2 q} s_{2}-1
\end{array}\right] .
$$

At the disease-free steady state, four eigenvalues of the Jacobian matrix are

$$
-\mu,-\mu, R_{1 q}-1, R_{2 q}-1 .
$$

All eigenvalues are negative if $R_{1 q}<1$ and $R_{2 q}<1$. Whenever $R_{1 q}>1$ or $R_{2 q}>1$, the steady state becomes unstable. Hence, the disease-free steady state is stable if and only if

$$
R_{1 q}<1 \text { and } R_{2 q}<1 .
$$


At the single-strain steady state of strain 1, the eigenvalues of the Jacobian matrix are

$$
-\mu-\sigma R_{1 q} i_{1 q}^{1}, \frac{-\left(\mu+R_{1 q} i_{1 q}^{1}\right) \pm \sqrt{\left(\mu+R_{1 q} i_{1 q}^{1}\right)^{2}+4 R_{1 q}^{2} s_{1 q}^{1} i_{1 q}^{1}}}{2} \text {, and } R_{2 q} S_{2 q}^{1}-1 .
$$

We see that all of these eigenvalues are negative if $R_{1 q}>1$ and $\left(R_{2 q}-1\right)-\sigma\left(R_{1 q}-1\right)<0$. Since the steady state is unstable if at least $\left(R_{2 q}-1\right)-\sigma\left(R_{1 q}-1\right)>0$, the single-strain steady state of strain 1 is stable if and only if

$$
R_{1 q}>1 \text { and }\left(R_{2 q}-1\right)-\sigma\left(R_{1 q}-1\right)<0 .
$$

At the single-strain steady state of strain 2, the stability conditions can be found in the similar way.

At the coexistent steady state, we use the Routh-Hurwitz criteria to derive stability conditions. We consider the characteristic equation of the Jacobian matrix as follows:

$$
\lambda^{4}+a_{1} \lambda^{3}+a_{2} \lambda^{2}+a_{3} \lambda+a_{4},
$$

where

$$
\begin{aligned}
& a_{1}=\left(\mu+R_{1 q} i_{1 q}^{*}+\sigma R_{2 q} i_{2 q}^{*}\right)+\left(\mu+R_{2 q} i_{2 q}^{*}+\sigma R_{1 q} i_{1 q}^{*}\right), \\
& a_{2}=\left(\mu+R_{1 q} i_{1 q}^{*}+\sigma R_{2 q} i_{2 q}^{*}\right)\left(\mu+R_{2 q} i_{2 q}^{*}+\sigma R_{1 q} i_{1 q}^{*}\right)+\left(R_{1 q}^{2} s_{1 q}^{*} i_{1 q}^{*}+R_{2 q}^{2} s_{2 q}^{*} i_{2 q}^{*}\right), \\
& a_{3}=R_{1 q}^{2} s_{1 q}^{*} i_{1 q}^{*}\left(\mu+R_{2 q} i_{2 q}^{*}+\sigma R_{1 q} i_{1 q}^{*}\right)+R_{2 q}^{2} s_{2 q}^{*} i_{2 q}^{*}\left(\mu+R_{1 q} i_{1 q}^{*}+\sigma R_{2 q} i_{2 q}^{*}\right) \\
& a_{4}=\left(1-\sigma^{2}\right) R_{1 q}^{2} R_{2 q}^{2} s_{1 q}^{*} i_{1 q}^{*} s_{2 q}^{*} i_{2 q}^{*} .
\end{aligned}
$$

Obviously, $a_{1}>0, a_{3}>0$, and $a_{4}>0$, when $i_{1 q}^{*}>0$ and $i_{2 q}^{*}>0$. After comparing and canceling similar terms of $a_{1} a_{2} a_{3}$ and $a_{3}^{2}+a_{1}^{2} a_{4}$, we have two terms left to be compared:

$$
\text { LHS: }\left(R_{1 q}^{2} s_{1 q}^{*} i_{1 q}^{*}-R_{2 q}^{2} s_{2 q}^{*} i_{2 q}^{*}\right)^{2}\left(\mu+R_{1 q} i_{1 q}^{*}+\sigma R_{2 q} i_{2 q}^{*}\right)\left(\mu+R_{2 q} i_{2 q}^{*}+\sigma R_{1 q} i_{1 q}^{*}\right) \text { from } a_{1} a_{2} a_{3},
$$

and

$$
\text { RHS: }-2 \sigma^{2} R_{1 q}^{2} R_{2 q}^{2} s_{1 q}^{*} i_{1 q}^{*} s_{2 q}^{*} i_{2 q}^{*}\left(\mu+R_{1 q} i_{1 q}^{*}+\sigma R_{2 q} i_{2 q}^{*}\right)\left(\mu+R_{2 q} i_{2 q}^{*}+\sigma R_{1 q} i_{1 q}^{*}\right) \text { from } a_{3}^{2}+a_{1}^{2} a_{4} .
$$

Whenever $i_{1 q}^{*}$ and $i_{2 q}^{*}$ are both positive, the LHS term is positive while the RHS term is negative. Hence,

$$
a_{1} a_{2} a_{3}>a_{3}^{2}+a_{1}^{2} a_{4}
$$

In case $i_{1 q}^{*}$ and $i_{2 q}^{*}$ have different signs, $a_{4}<0$. When $i_{1 q}^{*}$ and $i_{2 q}^{*}$ are negative, $a_{3}<0$ if $\left(\mu+R_{1 q} i_{1 q}^{*}+\right.$ $\left.\sigma R_{2 q} i_{2 q}^{*}\right)$ and $\left(\mu+R_{2 q} i_{2 q}^{*}+\sigma R_{1 q} i_{1 q}^{*}\right)$ are positive; $a_{1}<0$ if both of them are negative; and $a_{1} a_{2} a_{3}<$ $a_{3}^{2}+a_{1}^{2} a_{4}$ if they have different signs. Consequently, either $i_{1 q}^{*}$ or $i_{2 q}^{*}$ is negative, the coexistent steady state is unstable. Therefore, the coexistent steady state is stable if and only if $i_{1 q}^{*}>0$ and $i_{2 q}^{*}>0$, or

$$
R_{2 q}-1-\sigma\left(R_{1 q}-1\right)>0 \text { and } R_{1 q}-1-\sigma\left(R_{2 q}-1\right)>0 .
$$

Relating to the stability conditions in Theorem 3.1, Figure 2 shows the competition between two strains of influenza as functions of the basic reproductive numbers.

\subsection{The mean age at infection}

The mean age at infection or the mean time that a human remains susceptible is an important indicator of the disease prevalence [34]. Here, we study the mean age at infection of strain 1 by fixing the basic reproductive number of strain 2, varying the basic reproductive number of strain 1, and ignoring the small natural-death term in (2.1) when time is not rescaled. The mean age at infection of strain 2 can be studied in the similar way. We separate the mean age at infection into two cases:

1. in the absence of strain 2 
2. in the presence of strain 2

Proposition 3.2. Assume that $R_{1 q}>1$ and $R_{2 q}>1$.

1. The mean age at infection to strain 1 is absent when strain 2 persists $\left(R_{1 q}<\sigma\left(R_{2 q}-1\right)+1\right)$.

2. The mean age at infection to strain 1 is

$$
A_{2 q}=\frac{1}{\beta_{1} i_{1 q}^{*} N}=\frac{\left(1-\sigma^{2}\right)}{\mu_{0}\left[\left(R_{1 q}-1\right)-\sigma\left(R_{2 q}-1\right)\right]},
$$

when both strains exist or $\sigma\left(R_{2 q}-1\right)+1<R_{1 q}<\left(R_{2 q}-1\right) / \sigma+1$.

3. The mean age at infection to strain 1 is

$$
A_{1 q}=\frac{1}{\beta_{1} i_{1 q}^{1} N}=\frac{1}{\mu_{0}\left(R_{1 q}-1\right)}
$$

when strain 1 outcompetes strain 2 or $R_{1 q}>\left(R_{2 q}-1\right) / \sigma+1$.

Proof. This follows the stability conditions and the definition of the mean age at infection.

\subsection{Rate of invasion of the novel strain}

In this section instead of assuming that we have two strains of influenza co-circulating in the host population to study the long-term dynamics, we consider the situation that strain 1 is endemic first while strain 2 is absent in order to examine the invasion rate of strain 2 to invade a resident strain (strain $1)$.

Proposition 3.3. Assume that individuals are at the single-strain steady state of strain 1. The invasion rate of strain 2 is

$$
k_{q}=\frac{\left(R_{2 q}-1\right)-\sigma\left(R_{1 q}-1\right)}{\left.\left(1+\sigma\left(R_{1 q}-1\right)\right)\right)} .
$$

Proof. In the presence of isolation, the invasion dynamics of strain 2 at the beginning of invasion can be approximated by linearisation about the resident-strain steady state. Because $i_{2}^{\prime}(t)=R_{2 q} s_{2} i_{2}-i_{2}$,

$$
i_{2}(t)=C_{2} e^{\left(R_{2 q} s_{2}-1\right) t}=C_{2} e^{k_{q} t}
$$

where $C_{2}$ is a positive constant. Hence, $k_{q}=R_{2 q} s_{2 q}^{1}-1$.

\section{Numerical Studies}

We choose to show the numerical results when $R_{1}>R_{2}$. Parameter values can be found in Table 1 . If $R_{1}=3.5, R_{2}=3$, and $\sigma=0.75$, both strains coexist when $\theta<0.3333$; only strain 1 persists when $0.3333<\theta<0.7143$; and both strains die out when $\theta>0.7143$. Figure 3(a) shows the numerical result when $\theta=0.2$, so we have the coexistence of the strains. In Figure $3(\mathrm{~b}), \theta=0.5$, strain 2 is driven out by strain 1 . In Figure $3(\mathrm{c})$, we have $\theta=0.85$, so both strains die out from the host population.

In addition, we assume that the delay in isolation occurs for one month. Instead of starting the isolation at the beginning $(t=0)$, we introduce it one month later and compare it with the situation that the delay does not occur. We run the simulation with $\theta=0$ until one month and then continue running it but with $\theta=0.5$ after that and compare it with the result that we run the simulation with $\theta=0.5$ constantly from the beginning. The sample case that we further study here is from the previous result (see Figure 3(b)) where strain 1 is endemic and when $R_{1}=3.5, R_{2}=3$, and $\sigma=0.75$. The simulations were run until $t=5000$ in years but we only choose to show the results in some time intervals that the difference between the models are distinguished because the models are synchronized when time becomes very large. By starting with the same number of infectious individuals, we simulate the model in the situation that we 
have the delay in isolation to compare it with the situation that we start isolation at the beginning of the disease (see Figure 4). In Figure 4(a), we show the number of infectious individuals to strain 1 at the beginning of the disease. We can see that with the delay in isolation a high jump appears (or an outbreak) while the model without the delay produces the lower jumps. The high jumps continually appears but with the lower height when time increases (see Figure 4(b)) and finally the number of infectious diseases in both cases tends to $i_{1 q}^{1} N$ or $I_{1}$. For strain 2, it dies out quickly in the absence of isolation delay while it leads to one outbreak (a high jump) and then dies out in the presence of isolation delay (see Figure $4(\mathrm{c})$ ). From the result, we conclude that the delay in isolation leads to serious outbreaks.

By fixing the basic reproductive ratio of strain $2, R_{2 q}=3$, and $\sigma=0.8$, Figure 5 (a) shows that the host's mean age of infection is $A_{2 q}$ in (3.7) when $2.6<R_{1 q}<3.5$ or $A_{1 q}$ in (3.8) when $R_{1 q}>3.5$. In Figure 5(b), isolation extends the host's mean age of infection comparing with the system without intervention. Hence, isolation helps to increase the host's mean age of infection to the strain.

We consider the invasion rate of strain 2 by fixing $R_{1}=3, \sigma=0.8$, and $q_{0}=365.3$. Figure 6 shows the comparison of the invasion rate between with and without isolation. The results suggest that isolation helps to reduce the invasion rate of a new strain.

\section{Conclusion and discussion}

In this work, we introduce an isolation model for two co-circulating strains of influenza. The model is based on a status-based framework by [22] that individuals are categorized according to their immune status.

Based on this study, whether both strains of influenza die out or coexist, or only one strain of them persists, it depends on the basic reproductive number of each strain of influenza, cross-immunity between strains, and isolation rate (see Table 2). When isolation is delayed, our results suggest that the delay leads to higher jumps of the number of infectious individuals which represent more serious outbreaks than when there is no delay in isolation. Hence, launching isolation as soon as possible may help to control influenza effectively.

We study the mean age of infection to strain 1 when strain 2 is either absent or present. We show that the host's mean age of infection to strain 1 is extended (so hosts are unlikely to get infected) by isolation. By assuming that strain 1 is endemic in the host population, we study the invasion rate of a new strain. The model predicts that isolation helps to reduce the invasion rate of a new strain.

All in all, we hope that this study may help to understand the strain dynamics of influenza when a control strategy like isolation is applied.

Appendix

TABLE 1. Lists of parameters

\begin{tabular}{llll}
\hline Parameter & Description & Value & References \\
\hline$\mu_{0}$ & birth or death rate & $1 / 70$ & (estimated) \\
$\nu_{0}$ & recovery rate & $365 / 7$ & {$[22]$} \\
$\beta_{i}$ & transmission rate of strain $i$ & $R_{0}\left(\mu_{0}+\nu_{0}\right) / N$ & \\
$\sigma$ & cross-immunity coefficient (induced by infection) & $0.75-0.8$ & {$[22]$} \\
$q_{0}$ & rate of removing infectious individuals to isolation & $365 / 3$ (or varies) & (estimated) \\
$R_{0}$ & the basic reproductive number & 3 (or varies) & {$[31]$} \\
$N$ & the total size of population & 10000000 & (estimated) \\
$S_{i}$ & the number of susceptible individuals to strain $i$ & & \\
$I_{i}$ & the number of individuals infected with strain $i$ & & \\
$Q$ & the total number of isolated individuals & & \\
\hline
\end{tabular}


TABLE 2. Steady state chart for the isolation model.

\begin{tabular}{|c|c|c|c|c|}
\hline Basic reproductive numbers & $P_{q}^{0}$ & $P_{q}^{1}$ & $P_{q}^{2}$ & $P_{q}^{*}$ \\
\hline$R_{1 q}, R_{2 q}<1$ & stable & undefined & undefined & undefined \\
\hline $\begin{array}{l}R_{1 q}>1 \\
R_{2 q}<1+\sigma\left(R_{1 q}-1\right)\end{array}$ & unstable & stable & $\begin{array}{l}\text { unstable or } \\
\text { undefined }\end{array}$ & undefined \\
\hline $\begin{array}{l}R_{2 q}>1 \\
R_{1 q}<1+\sigma\left(R_{2 q}-1\right)\end{array}$ & unstable & $\begin{array}{l}\text { unstable or } \\
\text { undefined }\end{array}$ & stable & undefined \\
\hline $\begin{array}{l}R_{1 q}>1+\sigma\left(R_{2 q}-1\right) \\
R_{2 q}>1+\sigma\left(R_{1 q}-1\right)\end{array}$ & unstable & unstable & unstable & stable \\
\hline
\end{tabular}

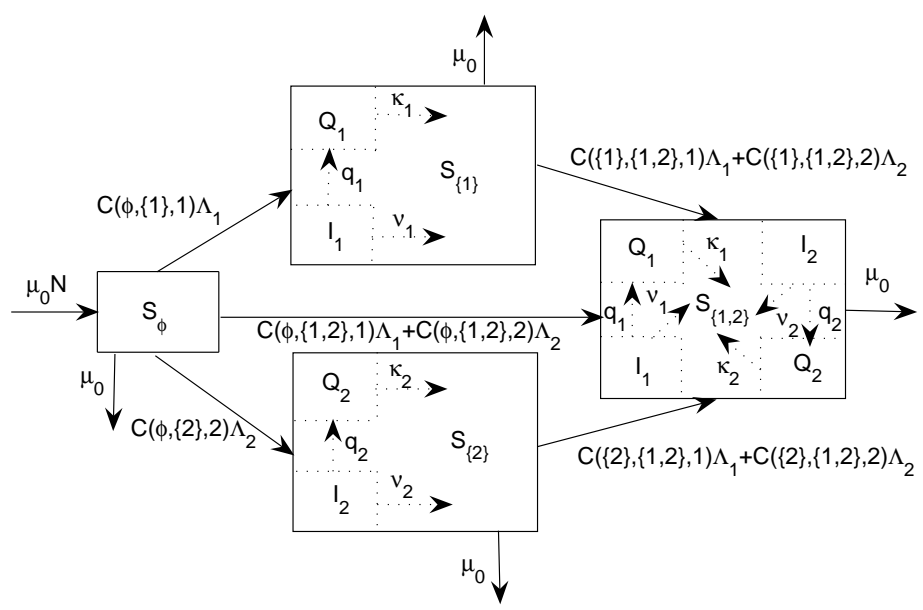

Figure 1. A flow diagram for the status-based model with isolation.

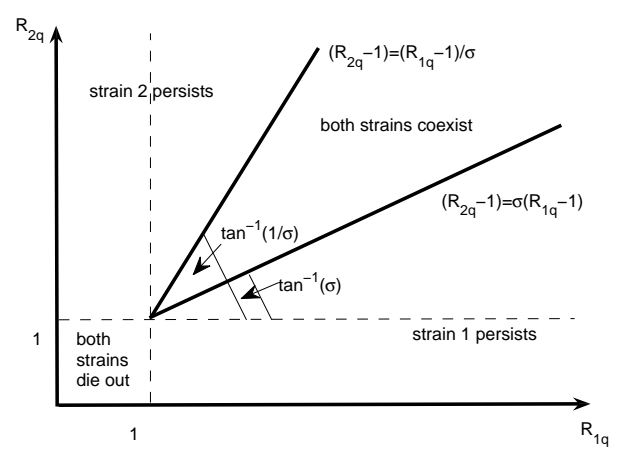

Figure 2. The competition between two strains of influenza as functions of the basic reproductive numbers when isolation is present. 

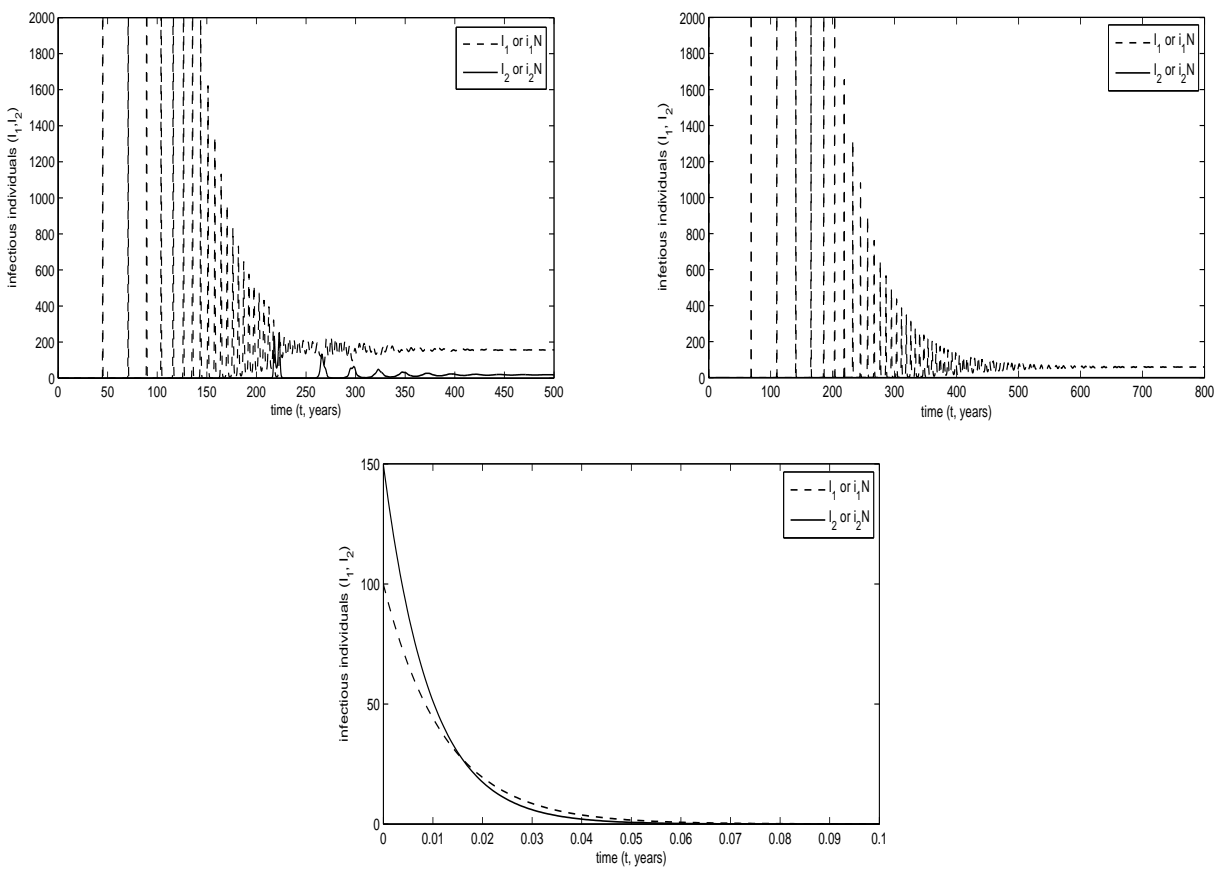

Figure 3. Numerical results when isolation is launched; (a) Coexistence of two strains occurs (b) Only strain 1 is endemic (c) Both strains go extinct.
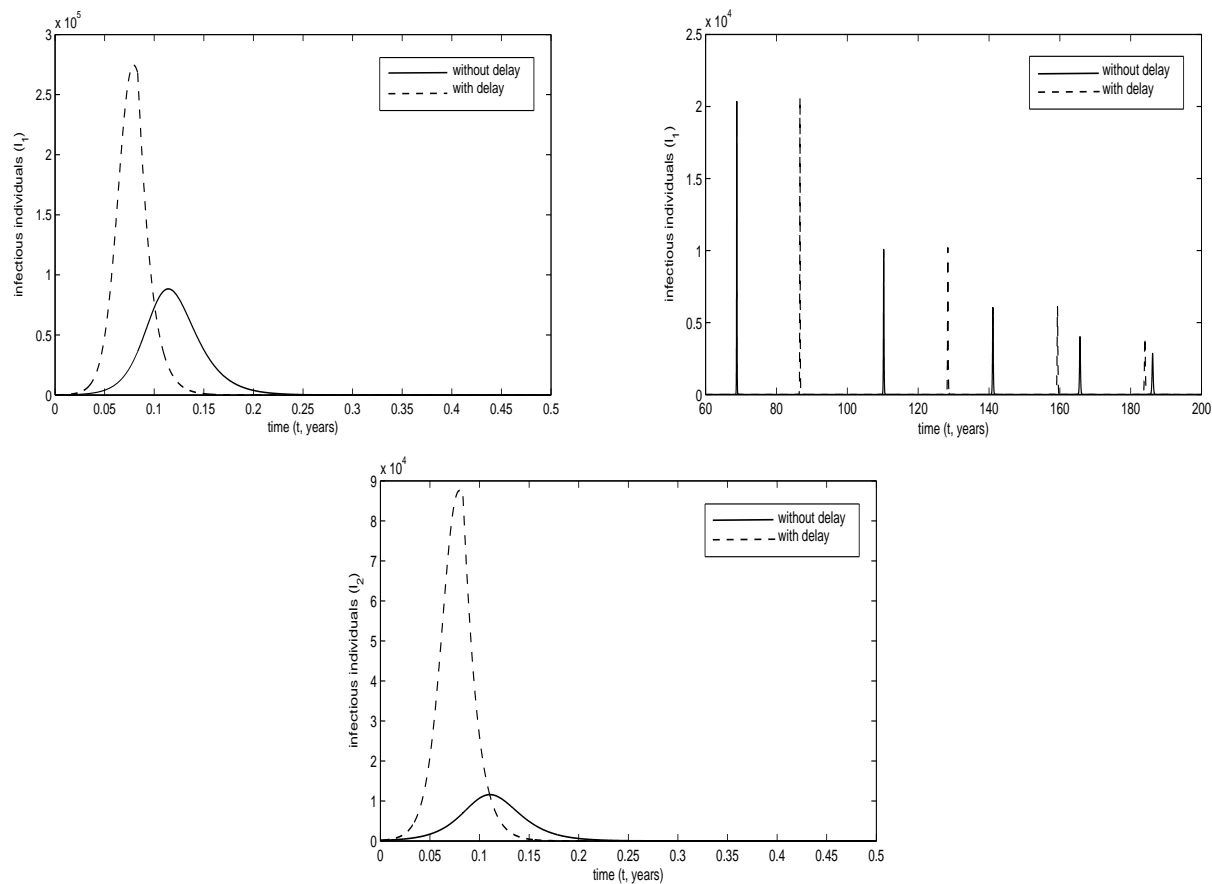

FiguRE 4. Numerical results when a delay in isolation occurs: (a) The dynamics of strain 1 at the onset of the disease: (b) The dynamics of strain 1 after the onset of the disease and when $t \in[60,200]$ (c) The dynamics of strain 2 

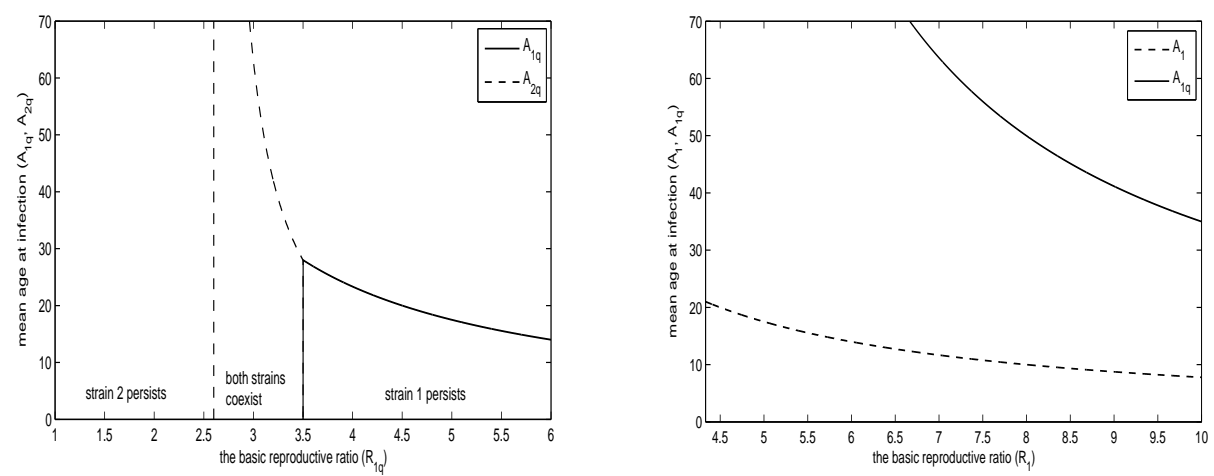

FiguRE 5. (a) The mean age of infection to strain 1 when $R_{2 q}=3, \sigma=0.8$, and $R_{1 q}$ is varied. (b) The comparison of $A_{1}$ when isolation is absent and $A_{1 q}$ when isolation is present (solid and dashed trace, respectively).

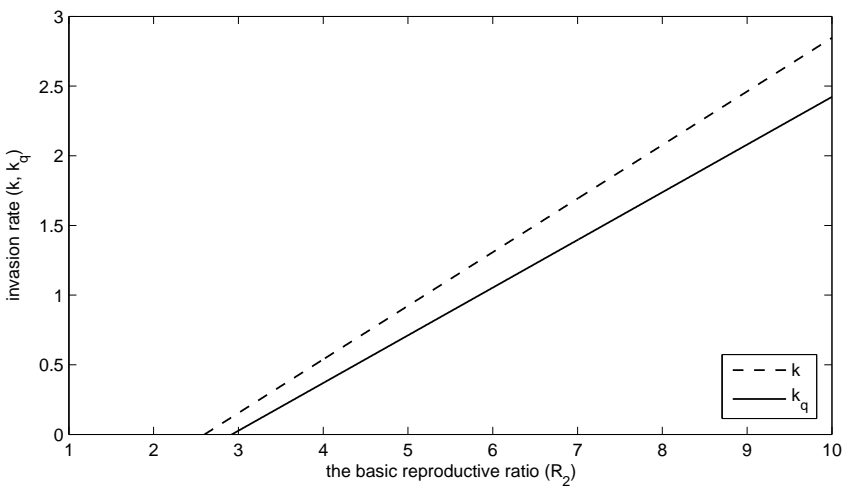

Figure 6 . The invasion rate when isolation is present (dashed trace) and absent (solid trace) $\left(R_{1}=3, \sigma=0.8, q_{0}=365 / 3\right)$. 
Acknowledgements. We would like to thank Dr. Shigui Ruan for very useful comments.

\section{References}

[1] R. Cattaneo, A. Schmid, D. Eschle, K. Baczko, V. T. Meulen, M. A. Billeter. Biased hypermutation and other genetic changes in defective measles viruses in human brain infections. Cell 55 (1988), 255-265.

[2] W. F. Carman, A. R. Zannetti, P. Karayiannis, J. Waters, G. Manzillo, E. Tanzi, A. J. Zuckermann, H. C. Thomas. Vaccine-induced escape mutant of hepatitis B virus. Lancet 336 (1990), 325-329.

[3] S. Sato, K. Suzuki, Y. Akahane, K. Akiyama, K. Yunomura, F. Tsuda, T. Tanaka, H. Okamoto, Y. Miyakawa, M. Mayumi. Hepatitis $B$ virus strains with mutations in the core promoter in patients with fulminant hepatitis. Ann. Internal Medicine 122 (1995), 241-248.

[4] W. N. Chen, C. J. Oon. Hepatitis B virus surface antigen (HBsAg) mutants in Singapore adult and vaccinated children with high anti-hepatitis B virus antibody levels but negative for HBsAg. J. Clin. Microbiol. 38 (2000), 2793-2794.

[5] J. J. Eron, P. L. Vernazza, D. M. Johnston, F. Seillier-Moiseiwitsch, T. M. Alcorn, S. A. Fiscus, M. S. Cohen. Resistance of HIV-1 to antiretroviral agents in blood and seminal plasma: Implications for transmission. AIDS 15 (1998), 181-189.

[6] G. D. Ebel, A. P. Dupuis, K. Ngo, D. Nicholas, E. Kauffman, S. A. Jones, D. Young, J. Maffei, P. Y. Shi, K. Bernard, L. D. Kramer. Partial genetic characterization of West Nile virus strains, New York State, 2000. Emerg. Infec. Dis. 7 (2000), 650-653.

[7] P. K. Cassiday, G. N. Sanden, K. Heuvelman, F. R. Mooi, K. M. Bisgard, T. Popovic. Polymorphism in Bordetella pertussis pertactin and pertussis toxin virulence factors in the United States. J. Infect. Dis. 182 (2000), 1402-1408.

[8] C. Weber, C. Boursaux-Eude, G. Coralie, V. Caro, N. Guiso. Polymorphism of Bordetella pertussis isolates circulating for the last 10 years in France, where a single effective whole-cell vaccine has been used for more than 30 years. J. Clin. Microbiol. 12 (2001), 4396-4403.

[9] S. Gupta, K. Trenholme, R. M. Anderson, K. P. Day. Antigenic diversity and the transmission dynamics of Plasmodium falciparum. Science 263 (1994), 961-963.

[10] P. Palese, J. F. Young. Variation of influenza A, B, and C viruses. Science 215 (1982), 1486-1474.

[11] R. G. Webster. Influenza: An emerging disease. Emerg. Infec. Dis. 4 (1998), 436-441.

[12] J. Davies, E. Grilli, A. Smith. Influenza A: infection and reinfection. J. Hyg.(Cambridge) 92, 125-127.

[13] H. Larson, D. Tyrrell, C. Bowker, C. Potter, G. Schild. Immunity to challenge in volunteers vaccinated with an inactivated current or earlier strain of influenza A(H3N2). J. Hyg. (Cambridge) 80, 243-248.

[14] A. L. Frank, L. H. Taber, J. M. Wells. Individuals infected with two subtypes of influenza A virus in the same season. J. Infect. Dis. 147 (1983), 120-124.

[15] T. Sonoguchi, H. Naito, M. Hara, Y. Takeuchi, H. Fukumi. Cross-subtype protection in humans during sequential, overlapping and/or concurrent epidemics caused by H3N2 and H1N1 influenza viruses. J. Infect. Dis. 151 (1985), 81-88.

[16] C. Castillo-Chavez, H. W. Hethcote, V. Andreasen, S. A. Levin, W. M. Liu. Epidemiological models with age structure, proportionate mixing, and cross-immunity. J. Math. Biol. 27 (1989), 233-258.

[17] S. Gupta, M. C. J. Maiden, I. M. Feavers, S. Nee, R. M. May, R. M. Anderson. The maintenance of strain structure in populations of recombining infectious agents. Nat. Med. 2 (1996), 437-442.

[18] V. Andreasen, J. Lin, S. A. Levin. The dynamics of cocirculating influenza strains conferring partial cross-immunity. J. Math. Biol. 35 (1997), 825-842.

[19] S. Gupta, N. Ferguson, R. M. Anderson. Chaos, persistence, and evolution of strain structure in antigenically diverse infectious agents. Science 280 (1998), 912-915.

[20] J. Lin, V. Andreason, S. A. Levin. Dynamics of influenza A drift:the linear three-strain model. Math. Biosci. 162 (1999), 33-51.

[21] J. R. Gog, J. Swinton. A Status-based Approach to Multiple Strain Dynamics. J. Math. Biol. 44 (2002), $169-184$.

[22] J. R. Gog, B. T. Grenfell. Dynamics and selection of many-strain pathogens. Proc. Natl. Acad. Sci. USA 99 (2002), 17209-17214.

[23] M. Kamo, A. Sasaki. The Effect of Cross-immunity and Seasonal Forcing in a multi-strain epidemic model. Physica D 165 (2002), 228-241.

[24] J. Lin, V. Andreasen, R. Casagrandi, S. A. Levin. Travelling waves in a model of influenza A drift. J. Theor. Biol. 222 (2003), 437-445.

[25] V. Andreasen, J. Lin, S. A. Levin. The dynamics of cocirculating influenza strains conferring partial cross-immunity. J. Math. Biol. 35 (1997), 825-842.

[26] M. F. Boni, J. R. Gog, V. Andreasen, F. B. Christiansen. Influenza drift and epidemic size: the race between generating and escaping immunity. Theor. Popul. Biol. 65 (2004), 179-191.

[27] O. Restif, B. T. Grenfell. Integrating life history and cross-immunity into the evolutionary dynamics of pathogens. Proc. R. Soc. B 273 (2006), 409-416.

[28] B. Adams, A. Sasaki. Cross-immunity, invasion and coexistence of pathogen strains in epidemiological models with one-dimensional antigenic space. Math. Biosci. 210 (2007), 680-699.

[29] P. Minayev, N. Ferguson. Improving the realism of deterministic multi-strain models: implications for modelling influenza A. J. R. Soc. Interface. 6 (2009) 509-518.

[30] C. Pease. An evolutionary epidemiological mechanism, with applications to type A influenza. Theor. Popul. Biol. 31 (1987) 422-452. 
[31] R. Casagrandi, L. Bolzoni, S. A. Levin, V. Andreasen. The SIRC model and influenza A. Math. Biosci. 200 (2006), 152-169.

[32] M. Nuno, Z. Feng, M. Martcheva, C. Castillo-Chavez. Dynamics of two-strain influenza with isolation and partial cross-immunity. SIAM J. Appl. Math. 65 (2005) 964-982.

[33] M. Nuno, G. Chowell, X. Wang, C. Castillo-Chavez. On the role of cross-immunity and vaccines on the survival of less fit flu-strains. Theor. Popul. Biol. 71 (2007) 20-29.

[34] M. J. Keeling, P. Rohani. Modeling Infectious Diseases in Humans and Animals. Princeton University Press, New Jersey, 2008.

[35] L. Edelstein-Keshet. Mathematical Models in Biology. Random House, New York, 1986 